\title{
District Heating Energy Generation Optimisation Considering Thermal Storage
}

\author{
Jonathan Reynolds, Muhammad Ahmad, and Yacine Rezgui
}

\begin{abstract}
Modern, decentralised, multi-energy vector districts have great potential to reduce primary energy consumption and greenhouse gas emissions. However, due to the complex nature of these systems, they require intelligent management to maximise their benefit. Therefore this study models the energy generation of a district heating plant for the purpose of hourly, operational optimisation. Crucially, non-linear, part-load efficiency curves, and minimum load percentages are included in the energy generation modelling as well as thermal energy storage. Due to the non-linearities, a metaheuristic, genetic algorithm, optimisation approach was utilised. The optimisation framework was applied to a case study district with three distinct thermal energy generation sources, a gas CHP, gas boiler, and biomass boiler. The optimisation controlled the load percentage of each technology as well as varying thermal storage capacity to minimise the cost of meeting the heat demand. The study found that compared to the current, rule-based approach, the optimisation achieved a significant cost saving of $12.7 \%$ without any thermal storage. As the thermal storage capacity was increased the potential cost saving was also shown to increase proportionally to $21.7 \%$ with $1000 \mathrm{kWh}$ of storage.
\end{abstract}

Index Terms-Smart Grid, District Heating, Genetic Algorithm, District Energy Management

\section{NOMENCLATURE}

$Q \quad$ Heat energy produced.

$P L \quad$ Part load percentage.

$C \quad$ Total capacity.

El Electrical energy produced.

$m \quad$ Mass of water.

$C p \quad$ Specific heat capacity of water.

$T \quad$ Water temperature.

$S \quad$ Storage percentage.

$\eta \quad$ Discharge/charge efficiency of storage tank.

Reln Relative efficiency of conversion unit.

Nomn Nominal efficiency of conversion unit.

$a, b, c \quad$ Empirical coefficients.

$F \quad$ Fuel required.

$P \quad$ Price per unit.

$\dot{Q} \quad$ Difference between supply and demand.

$V \quad$ Total net cost.

Subscripts

$t \quad$ Timestep.

Th Thermal.

El Electrical.

Max Maximum.

Day Day time price.

Night Night time price

The authors are with the BRE Institute of Sustainable Engineering at Cardiff University, corresponding email ReynoldsJ8@Cardiff.ac.uk.

$\begin{array}{ll}\text { Superscripts } & \\ \text { Unit } & \text { Specific generation technology. } \\ \text { S } & \text { Relating to storage. } \\ \text { DH } & \text { District heating. } \\ \text { CHP } & \text { Relating to CHP. } \\ \text { Bio } & \text { Relating to biomass. } \\ \text { GB } & \text { Relating to gas boilers. } \\ \text { RHI } & \text { From the renewable heat incentive. } \\ \text { Gas } & \text { From the gas network. }\end{array}$

\section{INTRODUCTION}

$\mathbf{I}$ MPROVING energy efficiency and hence reducing our energy consumption and greenhouse gas emissions is one of the key challenges of our time. The building sector is estimated to be responsible for around $40 \%$ of total energy consumption [1]. Therefore, it is critical to target this sector for energy efficiency improvements. A significant focus for both governments and researchers is the concept of the microgrid or smart grid. These concepts have developed due to the seismic shifts in energy generation planning in recent years coupled with rapid advancement in computational power and communication infrastructures. Increasingly, energy systems are diverging from large-scale, centralised, fossil fuel power plants in favour of smaller localised, often renewable based generation.

Whilst the implementation of smaller scale microgrids including renewable resources is promising, it does add additional complexity to the control and management of energy networks. Optimal control and dispatch of energy to a building or district requires maximum utilisation of uncontrollable renewable resources and hence a predictive control method is required. In order to extract the maximum benefit from renewable resources, energy storage capacity such as batteries, hot water tanks and power to gas are under consideration in research [2], [3], [4]. These can provide crucial flexibility in the energy network to enable optimal extraction of renewable energy.

District energy systems are further complicated by the increasingly integrated nature of previously distinct, multienergy vectors. For example, combined heat and power units (CHP) produce both electricity and heat, a heat pump produces useful heat energy from electricity input and a power to gas system can generate hydrogen or synthetic natural gas from excess electricity. Due to the intertwined nature of these systems, the optimal operation must consider each energy vector [5]. Due to the possible mix of energy generation within a district coupled with uncertainty of renewable supply 
and variable energy demand, district energy systems should no longer follow static, reactionary and rule-based control. Instead, computational resources should be utilised to apply optimisation techniques to this problem. This optimisation would not only be required at the design stage but should be applied at an hourly resolution due to constantly changing circumstances in a modern, multi-vector microgrid.

\section{A. Related Work}

Several studies have aimed to address the optimal dispatch of energy generation within a microgrid. For example. Staino, [6], developed a cooperative model predictive control (MPC) optimisation using mixed integer linear optimisation (MILP) to optimise the use of a shared heat pump. They found that due to the larger aggregated loads the heat pump had greater flexibility to shift load. MILP was also used in [7] to optimise shared, district level, energy generation and the use of electrical energy storage. Guan, [8], uses similar methods applied to a more complex district. The optimisation problem must satisfy both thermal and electrical demand through use of solar PV, batteries, a CHP and buying from the electrical grid. A more decentralised problem is considered in [9] from the point of view of the residential prosumer. A MILP optimisation problem was developed to maximise the profit from providing ancillary services to the electrical grid when equipped with PV and electrical energy storage.

As well as controlling the energy generation, some studies have attempted to control the energy demand of certain appliances. For example, [10], applied a mixed integer non-linear programming (MINLP) optimisation to a islanded microgrid scenario. The aim of the optimisation was to maximise the use of local renewable resources and apply load side management to minimise the cost of energy. Zhang, [11], developed a comprehensive smart house optimisation. It assumes a residential property with solar and wind energy, a CHP, thermal and electrical storage, an electric vehicle and smart loads. The developed MPC strategy saves around $40 \%$ of cost compared to a day-ahead optimisation strategy. A multi-stage MPC optimisation was produced in [12]. It considers uncontrollable and controllable load, electrical storage and appliance control. A medium term, 24-hour, optimisation is run every hour and then a second shorter term optimisation runs every 5 minutes to allow a reaction to prediction errors.

As is illustrated in this literature review, MILP is very common within microgrid energy dispatch studies. The papers described previously model energy conversion technologies in a simplistic manner, disregarding part load characteristics. Modelling these characteristics is vital to ensure a true optimal result and achieve higher average efficiency from district energy generation. Therefore, this study will aim to capture the non-linearities present within district generation by modelling part-load efficiency curves. To include this within an optimisation framework a genetic algorithm, GA, will be used due to its aptitude for handling non-linear optimisation. Furthermore, the effect of thermal energy storage capacity will be analysed through a district heating-based case study.

\section{Methodology}

This paper will aim to control the percentage load set points of district energy generation units along with the percentage of energy within a thermal storage tank. This section will describe the generic mathematical modelling used to model these district components as well as discuss the optimisation method used. Then the application of this methodology to the specific case study is described in Section 4. It is assumed that the forecasted energy demand for the next 24-hour period is known in advance. A $100 \%$ forecasting accuracy is impossible in reality but it is envisaged advanced AI prediction methodologies could be applied, such as those developed in [13], [14], [15], [16] to achieve reasonably accurate demand forecasts.

\section{A. Mathematical Modelling of District Components}

This section will discuss the modelling of heat and electrical generation units. The equations displayed in this section are generic and could be applied to a wide range of possible technologies such as boilers, heat pumps, or combined heat and power (CHP) units. From the part load percentage set by the district control system the energy produced (either heat, electricity or both) can be calculated by:

$$
\begin{aligned}
& Q_{t}^{U n i t}=P L_{t}^{U n i t} \cdot C_{T h}^{U n i t} \\
& E l_{t}^{U n i t}=P L_{t}^{U n i t} \cdot C_{E l}^{U n i t}
\end{aligned}
$$

Where $Q_{t}^{U n i t}$ is the heat produced by a specific conversion technology, Unit, at a specific timestep, t. $P L$, is the part load percentage and $C_{T h}^{U n i t}$ is the thermal capacity of the conversion technology. $E l_{t}^{U n i t}$ is the electricity produced, and $C_{E l}^{U n i t}$ is the electrical capacity of the unit.

In this study the thermal storage will be modelled relatively simply as a percentage of its maximum load. This uses the simplifying assumption that there is no heat loss from the storage tank to the environment, that the temperature throughout the tank is constant and the district supply temperature is constant. Following these assumptions, the maximum amount of energy within a thermal storage tank is:

$$
C_{T h}^{S}=m \cdot C p \cdot\left(T_{M a x}^{S}-T^{D H}\right)
$$

Where, $Q_{\text {Max }}^{S}$, is the maximum heat energy stored within the tank, $m$, is the mass of water, $C p$, is the specific heat capacity of water taken as a constant, $T_{\text {Max }}^{S}$, is the maximum temperature of the tank, and $T^{H}$ is the supply temperature to the district heating network. From this equation it is evident that the only factor determining the amount of energy in the storage tank is the tank temperature, thus tank temperature can easily be translated to percentage of tank capacity and vice versa.

The net heat supply or draw from the storage tank at each hour is calculated using equation (4), requiring the initial storage tank conditions.

$$
Q_{t}^{S}=\left(S_{t}-S_{t-1}\right) \cdot \eta^{s} \cdot C_{T h}^{S}
$$

Where $Q_{t}^{S}$ is the heat supply or demand from the storage at timestep $t$, and $S$ is the percentage charge of the thermal 
storage tank and $\eta^{S}$ is the charging and discharging efficiency of the tank.

The raw fuel consumption of the energy conversion units are calculated using equations (5) and (6). These crucially take into consideration the part load efficiency curves of the energy conversion units using an empirical polynomial regression equation. This captures the characteristic drop is efficiency at low power outputs.Similar part load modelling approaches have been used in [17], [18], [19]. The empirical constants would need to be derived experimentally on site or provided in manufacturer data.

$$
\begin{aligned}
& \operatorname{Rel} \eta_{t}^{U n i t}=a \cdot\left(P L_{t}^{U n i t}\right)^{2}+b \cdot\left(P L_{t}^{U n i t}\right)+c \\
& F_{t}^{U n i t}=\frac{Q_{t}^{U n i t}}{N o m \eta^{U n i t} \cdot \text { Rel }_{t}^{\text {Unit }}}
\end{aligned}
$$

Where $R e l \eta_{t}^{U n i t}$ is the relative efficiency of the conversion unit at timestep $t$ due to the part load characteristics and Nom $\eta^{\text {Unit }}$ is the nominal efficiency given in manufacturer datasheets which remains constant. $F$ represents the raw fuel consumption (e.g. gas or biomass) and $a, b$ and $c$ are empirical constants.

Note that in this paper the district heating network itself is not modelled, this requires a number of simplifying assumptions. There are no thermal losses within the distribution network and there are no thermal lag characteristics meaning that heat is instantly delivered to the demand. In theory, these factors could be taken into consideration when predicting the demand profile. Instead of predicting the demand at a building level and aggregating the sum, the demand prediction would be the profile the district heating system is required to produce accounting for the thermal lag and network losses. If using AI machine learning methods (such as those referenced at the beginning of this section) that were trained on data from the central energy hub this would be intrinsically captured in the data.

\section{B. Optimisation Methodology}

In this study, a genetic algorithm, GA, will be applied to generate the optimal schedule of energy generation and storage management. GAs have previously been overlooked in the literature in favour of convex programming approaches such as MILP. However, these are required to make several simplifications or adjustments to the problem to remove all non-linearities such as part-load factors. On the contrary, a GA is a metaheuristic global optimisation approach inspired by the biological process of natural selection. Whilst metaheuristic optimisations cannot guarantee achieving an absolute optimal solution they are adept and handling non-linearities and have been applied to several other building-related optimisation problems with high success.

A GA randomly generates a population of feasible solutions with a chromosome length equal to the number of decision variables. Each individual solution within the population is assessed using a pre-defined fitness function. From this, each solution is ranked in relation to its applicability to the problem. Once the population has been ranked a selection function

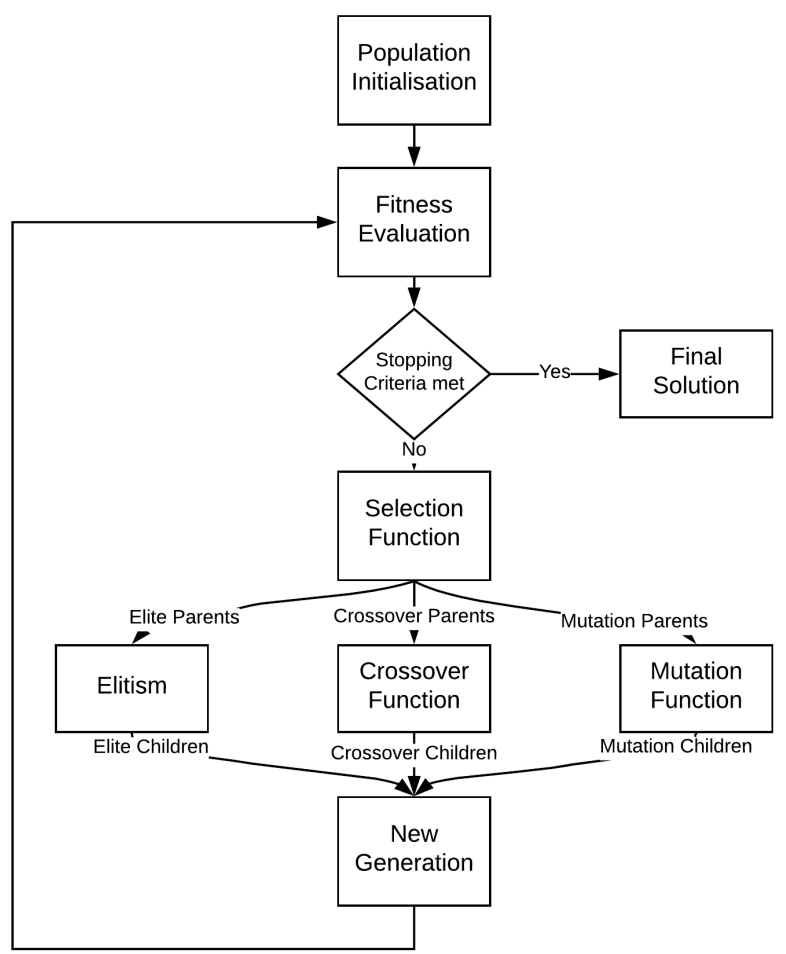

Fig. 1. Generic genetic algorithm procedure

is applied to select individuals to crossover or mutate. The crossover stage selects two parent solutions from the current population and combines the chromosomes of each parent to produce a child solution. The exact mechanism by which the two parents are combined is controlled by the crossover function. The mutation stage provides the potential for any chromosome within an individual solution to randomly mutate to any feasible solution. This guarantees the diversity of the population and to ensure the optimisation does not get stuck in local optima. Furthermore, some GAs will use elitism. This function takes the top percentage of solutions and automatically copies them into the next generation. The combined child solutions resulting from crossover, mutation and elitism form the next generation of solutions. The algorithm iterates to produce new generations until the GA stopping criteria has been met. The stopping criteria can relate to the maximum time, maximum number of generations or minimum deviation in the fitness over time. Once complete the individual with the best fitness is selected as the optimal solution. A schematic of the GA procedure is provided in Fig. 1.

\section{CAse Study Application}

The case study for which the developed methodology was applied is a modern eco-district based in Ebbw Vale, UK. The district is comprised of a secondary school, a higher education college, a leisure centre, an office building and a car park. These buildings are supplied with heat from an energy centre via a district heating network. The energy centre includes a $400 \mathrm{~kW}_{\text {th }}\left(375 \mathrm{~kW}_{\mathrm{el}}\right) \mathrm{CHP}$, two $499 \mathrm{~kW}_{\text {th }}$ biomass boilers and four $1600 \mathrm{~kW}_{\text {th }}$ gas boilers. Currently the site has two $17.5 \mathrm{~m}^{3}$ hot water buffer thanks although these are not used actively by 
TABLE I

STATIC INFORMATION REGARDING THE CASE STUDY DISTRICT

\begin{tabular}{ll}
\hline Symbol & Value \\
\hline$C_{T h}^{C H P}$ & $400 \mathrm{~kW}$ \\
$C_{E l}^{C H} P$ & $375 \mathrm{~kW}$ \\
$C_{T h}^{B i o}$ & $998 \mathrm{~kW}$ \\
$C_{T h}^{G B}$ & $6400 \mathrm{~kW}$ \\
$N o m \eta_{T h}^{C H P}$ & 0.40 \\
$N o m \eta_{E l}^{C H P}$ & 0.35 \\
$N o m \eta_{T h}^{B i o}$ & 0.82 \\
$N o m \eta_{T h}^{G B}$ & 0.67 \\
$P_{D a y}^{E l}$ & $10.9491 \mathrm{p} / \mathrm{kWh}$ \\
$P_{N i g h t}^{E l}$ & $7.2629 \mathrm{p} / \mathrm{kWh}$ \\
$P^{R H I}$ & $2.960 \mathrm{p} / \mathrm{kWh}$ \\
$P^{G a s}$ & $1.837 \mathrm{p} / \mathrm{kWh}$ \\
$P^{B i o}$ & $20.50 \mathrm{p} / \mathrm{kg}$ \\
\hline
\end{tabular}

the control system as storage, simply as mixing vessels before injection into the main district heating network. The electricity produced by the CHP is sold directly to the nearby higher education college for the same price it would otherwise buy electricity from the national grid (note there are two prices for day and night). The energy centre also receives income from biomass generation due to the UK governments renewable heat incentive (RHI) which gives a subsidy based on the number of $\mathrm{kWhs}$ produced from biomass. Additional static information in regard to the case study is provided in Table I.

\section{A. Fitness Function Formulation}

The optimisation procedure developed in this paper is outlined schematically in Fig. 2 and developed in the MATLAB environment. As this figure demonstrates, the inputs to the fitness function, (and decision variables of the GA) are the percentage loads of the CHP, biomass and thermal storage at each hour for 24-hours. Note that the percentages are related to the total installed capacity rather than one specific boiler. So, for example a $50 \%$ capacity in relation to the Biomass decision variable would mean one out of two boilers is turned on to $100 \%$ of its rated capacity. From these decision variables, the heat generated each hour is calculated using equations (1) and (4). The generated heat is aggregated and compared to the predicted heat demand. The mismatch, $\dot{Q}_{t}$, between heat generated and demand required for each hour is calculated using equation (7):

$$
\dot{Q}_{t}=\left(Q_{t}^{C H P}+Q_{t}^{\text {Bio }}+Q_{t}^{S}\right)-Q_{t}^{\text {Demand }}
$$

During any hour in which there is a heat deficit, the gas boilers are automatically set to meet that deficit. The fitness function was arranged in this way for several reasons. Firstly, modelling the constraint of supply matching demand in a GA is difficult, you either apply harsh penalty functions based on the deficit or ensure all solutions are feasible. By using our described method, we ensure that heat demand is always met removing the need to handle this constraint (note that

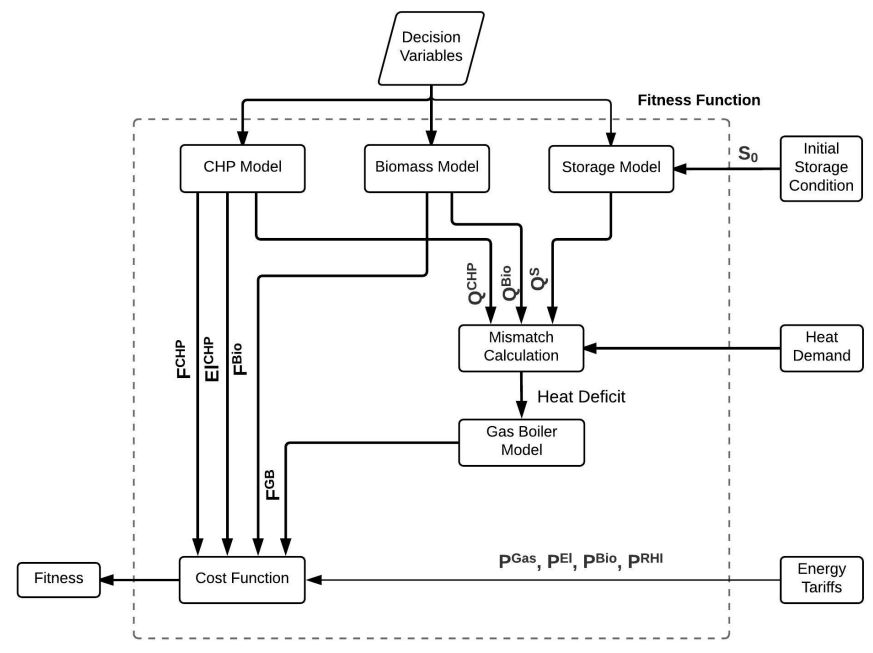

Fig. 2. Fitness Calculation Procedure

oversupply is not explicitly punished by the optimisation and the ability to dump load is assumed). Secondly, it removes the requirement for an additional 24 variables which is an important consideration for a GA. While the gas boilers are not explicitly controlled by the GA as decision variables, their influence will be implicit in the resulting fitness evaluation. The gas boilers were selected for this role due to the specific configuration of the case study district. In this case study, the gas boilers were intentionally oversized to provide backup generation and crucial flexibility to system.

Finally, once the heat from each source has been calculated the primary energy consumed to produce it is calculated. The relative efficiency of each generation technology in relation to the percentage load is displayed in Fig. 3. These profiles were generated from analysis of site conditions as well as manufacturer data. The number of generation units is reflected in the 'jaggedness' of the part load curves. For example, the biomass relative efficiency reaches 1.0 at $50 \%$ as one biomass boiler is working at full capacity. However, the relative efficiency then drops when above $50 \%$ as both biomass boilers then operate at part loads and hence lower efficiency. A similar trend is displayed for the gas boilers however, there are three discontinuities rather than one as there are four gas boilers in total. The relative efficiency based on these curves is used to calculate the instantaneous actual efficiency of each technology at each timestep from which the fuel consumption can be calculated from equation (6). Finally, the fitness of each solution is chosen to be the net cost of energy. This is calculated by summing the cost of the different fuels, minus the income from the sale of the electricity from the CHP and the income from the RHI as shown in equation (8):

$V=\sum_{t=1}^{24}\left(F_{t}^{G B}+F_{t}^{C H P}\right) \cdot P^{G a s}-\left(E l_{t}^{C H P} \cdot P^{E l e c}\right)-\left(Q_{t}^{B i o} \cdot R H I\right)$ 


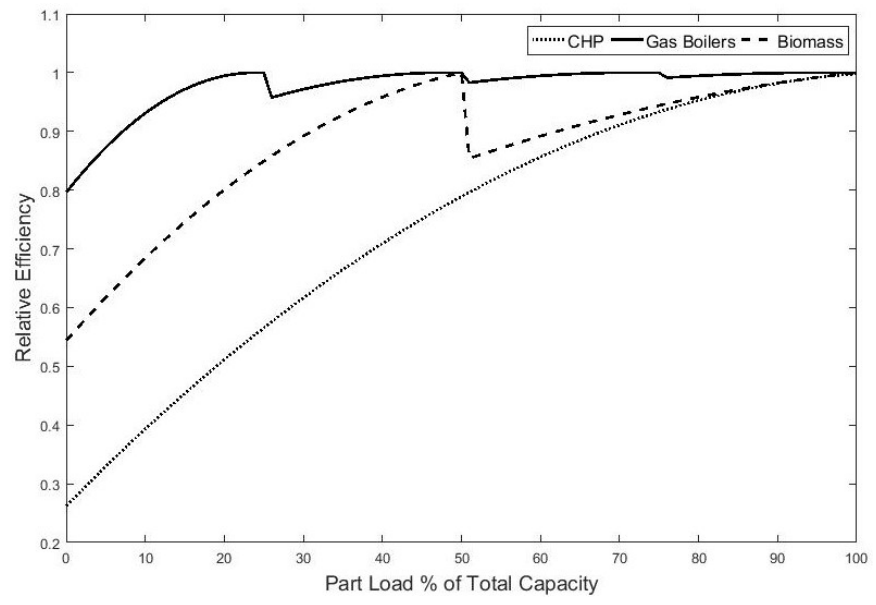

Fig. 3. Part load characteristics of the generation technology

\section{B. Genetic Algorithm Configuration}

As well as the part load factors this study also models the minimum load characteristics of the generation units. In this case study, the CHP cannot operate at conditions lower than $70 \%$ of its rated output and the biomass boilers cannot operate less than $50 \%$ of their rated output. To capture this within the GA structure, custom functions were required as the default MATLAB creation and mutation functions would lead to infeasible solutions due to the non-continuous nature of the decision variables (e.g. allowable solutions for the CHP are $[0,70-100])$. The custom creation function generated a random population of feasible solutions. To achieve this, 24 random integers between 69 and 100 for the CHP chromosomes and integers between 24 and 100 for the biomass chromosomes were generated. Chromosomes selected as 69 or 24 respectively were then overwritten to 0 . The final 24 chromosomes, relating to the thermal storage, are simply any integer between 0 and 100. The MATLAB crossoverscattered was applied as the crossover function as this simply recombines existing chromosomes from two parent solutions meaning that if parent solutions are feasible then child solutions would also be feasible. However, a custom mutation function is also required to ensure feasibility. A constant mutation rate of 0.05 is set. For each chromosome a random number between 0 and 1 is generated, if this is less than the mutation rate, the chromosome mutates to a random feasible number generated in an identical method to that described in the creation function. Additional GA parameters include a population size of 200 , maximum number of generations as 7200 and a function tolerance of 1x10-7. In all cases described in Section 4 the optimisation terminated due to the average change in fitness value being lower than the function tolerance, showing that the GA had converged.

\section{Optimisation Objectives}

The optimisation procedure described in this case study will aim to minimise the cost of meeting the energy demands of the district. To illustrate the benefits of the optimisation procedure, results will be compared to a baseline scenario which uses the existing rule-based strategy of the facility manager. This follows a generation priority order where the CHP is used initially to meet the base load, then the biomass boilers are turned on if load exceeds the CHP supply and finally the gas boilers provide the additional flexibility at high load periods. Note that in the baseline scenario, thermal storage is not actively used. Furthermore, the effect of varying thermal storage capacity on energy cost will be assessed.

\section{OPTIMISATION RESUlTS}

Initially the baseline scenario is compared to the optimisation result with no storage active storage capacity included. The two energy generation profiles are displayed in Fig. 4. Initially studying the baseline, the priority order is very clear, however, hour 22 shows a period in which the additional demand does not exceed the minimum load threshold of $25 \%$ of biomass capacity and hence the gas boilers are used as the second priority. Even without any storage capacity the optimisation makes several changes to the generation profile. Most obvious is the CHP turned on at full capacity even when the demand is lower. This is due to the relatively high sale price of electricity resulting in profit per $\mathrm{kWh}$ of gas consumed. Secondly at hours 20 and 21, the biomass load percentage is set to $50 \%$ and the excess demand is met by the gas boilers instead of solely by the biomass boilers. This shows the impact of including part load characteristics in an energy generation optimisation. The optimisation strategy prioritises running one biomass boiler at $100 \%$ relative efficiency as opposed to turning on a second biomass boiler and reducing the efficiency due to part load characteristics. Overall, these changes lead to a cost saving of $12.77 \%$ or $£ 36.42$ over the day without any storage capacity.

As demonstrated in Fig. 5, there is a clear relationship between increasing the thermal storage capacity and achieving greater daily energy savings, however this does appear to level out towards $1000 \mathrm{kWh}$. To understand the decisions the optimisation takes with the additional flexibility thermal energy storage provides, the energy generation profile with $800 \mathrm{kWh}$ of storage is shown in Fig. 6. Note that throughout the different storage capacities the key trends of storage deployment are similar but more exaggerated at higher capacities. Here we can see the optimisation choosing to store the excess heat generation from the CHP in the early hours until fully charged. This stored heat is then strategically deployed throughout the day to minimise generation via the gas boiler, for example at hours 7 and 18 to 22 . This behaviour results in a 5\% reduction in the gas boiler consumption. Furthermore, throughout the test day the biomass boilers operate with an average relative efficiency of nearly $100 \%$. These changes combined lead to a $21.7 \%$ (£61.90) reduction in daily energy cost whilst still meeting the demand required from the consumer.

This test case scenario has demonstrated that static, rulebased, priority controls can be improved upon by introducing a dynamic, predictive, optimisation-based controller. It is envisaged by the authors that predictive control, such as that presented in this paper, could be run multiple times every day in a sliding window, model predictive control method. 

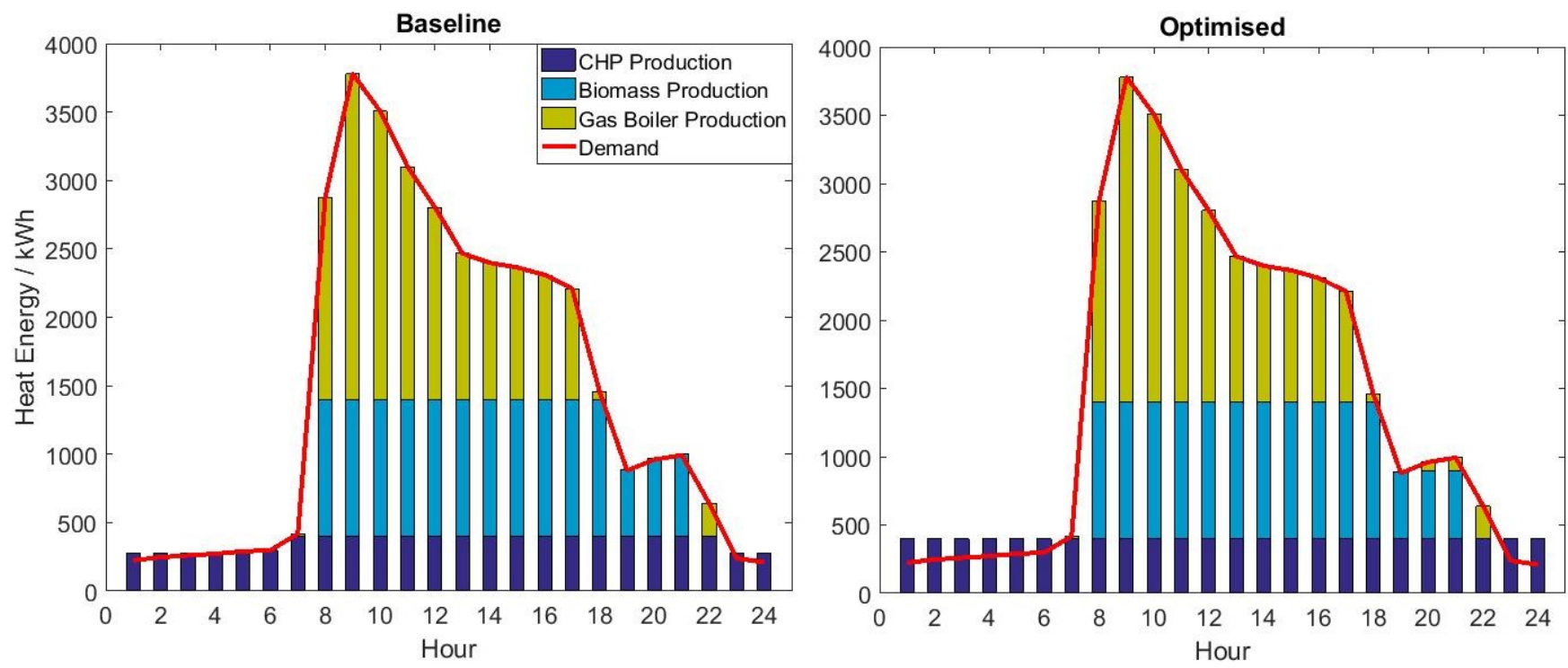

Fig. 4. Baseline vs Optimised heat generation strategies with no thermal storage

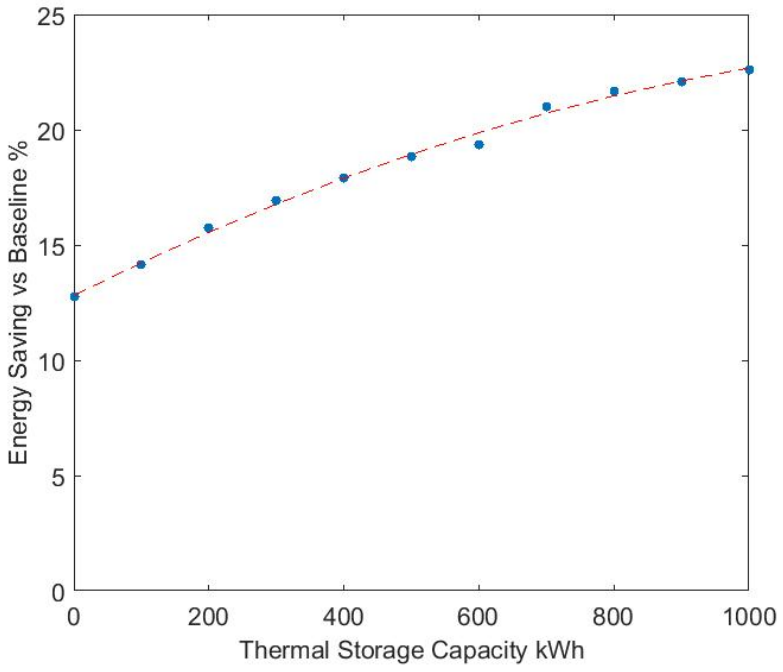

Fig. 5. Optimised energy savings vs thermal storage capacity

This means that the optimiser would run around every hour with a 24-hour optimisation horizon. This would allow the control strategy to adapt to feedback from the system and receive updated, more accurate demand forecasts. In future work, the authors aim to develop an improved district model that considers additional generation factors such as start-up and shutdown periods and costs. This will be demonstrated in a more complex case study district that will more explicitly factor in electricity demands as well as a power-to-gas system and uncontrollable renewable resources such as solar or wind power.

\section{CONCLusion}

The presented paper has generically modelled the energy generation capacity of a decentralised district energy system. This was used in conjunction with an optimisation framework

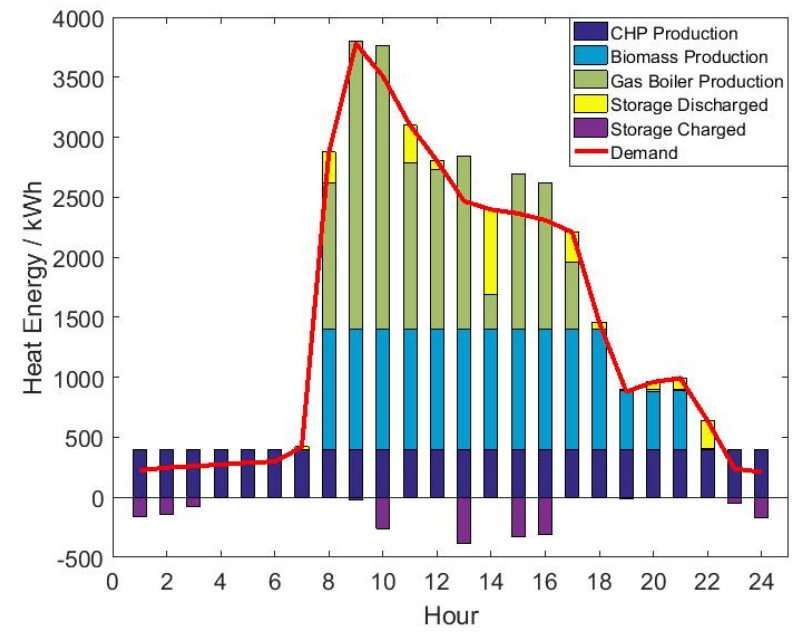

Fig. 6. Optimised energy generation profile with $800 \mathrm{kWh}$ of thermal energy storage

using a genetic algorithm with the ability to handle continuous part load generation efficiencies and minimum generation loads. The procedure was applied to a case study, eco-district located in Ebbw Vale, UK. The district contained three generation technologies, a CHP, gas boilers and biomass boilers supplying heating for five buildings via a district heating network. As well as the generation technologies, thermal energy storage has been modelled to provide crucial flexibility to the system.

The case study results contained demonstrate the potential energy savings that could be achieved by moving from a rulebased control strategy, to an optimisation-based, predictive control method. Without any storage capacity modelled the optimisation makes significant energy cost savings of $12.7 \%$ by increasing CHP production and recognising decreases in efficiency at specific loads. The results also demonstrate the 
positive relationship between thermal storage capacity and reduction in energy cost. Maximum utilisation of storage capacity demonstrated by the optimisation procedure effectively limits the number of hours in which the gas boilers are used. This resulted in a $21.7 \%$ reduction in cost when $1000 \mathrm{kWh}$ of thermal storage was available.

\section{ACKNOWLEDGMENT}

The authors would like to acknowledge the financial support of EPSRC (Engineering and Physical Sciences Research Council) and BRE (Building Research Establishment) as well as the European Commission as part of the Horizon2020 THERMOSS (Project Id: 723562) and PENTAGON (Project Id: 731125) projects.

\section{REFERENCES}

[1] J. Reynolds, Y. Rezgui, and J.-L. Hippolyte, "Upscaling Energy Control from Building to Districts: Current Limitations and Future Perspectives," Sustainable Cities and Society, pp. 1-15, may 2017. [Online]. Available: http://linkinghub.elsevier.com/retrieve/pii/S221067071730135X

[2] Y. Maniyali, A. Almansoori, M. Fowler, and A. Elkamel, "Energy Hub Based on Nuclear Energy and Hydrogen Energy Storage," Industrial \& Engineering Chemistry Research, vol. 52, pp. 7470-7481, 2013. [Online]. Available: http://pubs.acs.org/doi/abs/10.1021/ie302161n

[3] T. Chen, "Real-time predictive supervisory operation of building thermal systems with thermal mass," Energy and Buildings, vol. 33, no. 2, pp. 141-150, 2001.

[4] B. P. Koirala, E. Koliou, J. Friege, R. A. Hakvoort, and P. M. Herder, "Energetic communities for community energy: A review of key issues and trends shaping integrated community energy systems," Renewable and Sustainable Energy Reviews, vol. 56, pp. 722-744, 2016. [Online]. Available: http://dx.doi.org/10.1016/j.rser.2015.11.080

[5] X. Liu and P. Mancarella, "Modelling, assessment and Sankey diagrams of integrated electricity-heat-gas networks in multi-vector district energy systems," Applied Energy, vol. 167, pp. 336-352, 2016.

[6] A. Staino, H. Nagpal, and B. Basu, "Cooperative Optimizationof Building Energy Systems in an Economic Model Predictive Control Framework," Energy and Buildings, vol. 128, pp. 713-722, 2016 [Online]. Available: http://dx.doi.org/10.1016/j.enbuild.2016.07.009

[7] A. Parisio, C. Wiezorek, T. Kyntäjä, J. Elo, and K. H. Johansson, "An MPC-based Energy Management System for Multiple Residential Microgrids," in IEEE International Conference on Automation Science and Engineering (CASE), Gothenburg, Sweden, 2015.
[8] X. Guan, Z. Xu, and Q.-s. Jia, "Energy-Efficient Buildings Facilitated by Microgrid," IEEE Transactions on Smart Grid, vol. 1, no. 3, pp. 243-252, dec 2010. [Online]. Available: http://ieeexplore.ieee.org/document/5628267/

[9] C. Clastres, T. T. Ha Pham, F. Wurtz, and S. Bacha, "Ancillary services and optimal household energy management with photovoltaic production," pp. 55-64, 2010.

[10] M. Marzband, A. Sumper, A. Ruiz-Alvarez, J. Luis Dominguez-Garcia, and B. Tomoiaga, "Experimental validation of a real time energy management system for microgrids in islanded mode using a local day-ahead electricty market and MINLP," Energy Conversion and Management, vol. 76, pp. 365-376, 2013.

[11] Y. Zhang, T. Zhang, R. Wang, Y. Liu, and B. Guo, "Optimal operation of a smart residential microgrid based on model predictive control by considering uncertainties and storage impacts," Solar Energy, vol. 122, pp. 1052-1065, 2015. [Online]. Available: http://linkinghub.elsevier.com/retrieve/pii/S0038092X15005782

[12] J. K. Gruber, F. Huerta, P. Matatagui, and M. Prodanovic, "Advanced building energy management based on a two-stage receding horizon optimization," Applied Energy, vol. 160, pp. 194-205, 2015.

[13] Y. T. Chae, R. Horesh, Y. Hwang, and Y. M. Lee, "Artificial neural network model for forecasting sub-hourly electricity usage in commercial buildings," Energy and Buildings, vol. 111, pp. 184-194, jan 2016. [Online]. Available: http://linkinghub.elsevier.com/retrieve/pii/S0378778815304102

[14] B. Yuce, H. Li, Y. Rezgui, I. Petri, B. Jayan, and C. Yang, "Utilizing artificial neural network to predict energy consumption and thermal comfort level: An indoor swimming pool case study," Energy and Buildings, vol. 80, pp. 45-56, sep 2014. [Online]. Available: http://linkinghub.elsevier.com/retrieve/pii/S0378778814003788

[15] M. W. Ahmad, M. Mourshed, and Y. Rezgui, "Trees vs Neurons: Comparison between Random Forest and ANN for high-resolution prediction of building energy consumption," Energy and Buildings, apr 2017. [Online]. Available: http://linkinghub.elsevier.com/retrieve/pii/S0378778816313937

[16] M. Benedetti, V. Cesarotti, V. Introna, and J. Serranti, "Energy consumption control automation using artificial neural networks and adaptive algorithms: Proposal of a new methodology and case study," Applied Energy, vol. 165, pp. 60-71, 2016.

[17] P. Raman and N. Ram, "Performance analysis of an internal combustion engine operated on producer gas, in comparison with the performance of the natural gas and diesel engines," Energy, vol. 63, pp. 317-333, 2013.

[18] M. A. Meybodi and M. Behnia, "Optimum sizing of the prime mover in a medium scale gas turbine chp system," Journal of Engineering for Gas Turbines and Power, vol. 133, no. 11, p. 112001, 2011.

[19] H. Li, R. Nalim, and P.-A. Haldi, "Thermal-economic optimization of a distributed multi-generation energy systema case study of beijing," Applied Thermal Engineering, vol. 26, no. 7, pp. 709-719, 2006. 